\title{
Teamführung
}

\section{Mit wenigen Schritten zu mehr Effizienz in der Praxis}

\author{
Studien zufolge leistet ein gut eingespieltes Praxisteam einen erheblichen Beitrag \\ zum Erfolg einer Arztpraxis. Gut eingespielt ist ein Team aber nur, wenn die Führungs- \\ spitze den notwendigen Rahmen dafür setzt. Das Problem vieler Ärzte: Sie sind auf \\ diese Aufgabe - die Entwicklung von Mitarbeitern zu einem leistungsfähigen Team - \\ zu Beginn ihrer Tätigkeit meist nicht vorbereitet.
}

D er häufigste Fehler steckt bereits in der Annahme, dass die Praxismitarbeiter die gerechte Verteilung von Aufgaben oder auch Konflikte „wie Erwachsene untereinander selbst regeln“. Bei Konflikten ist es zum Beispiel besonders wichtig, sich als Vorgesetzter frühzeitig einzuschalten. Denn Streit im Praxisteam kann schnell eskalieren und zu einer Frontenbildung oder Spaltung des Teams führen. Studien zufolge beschäftigen sich die Konfliktbeteiligten etwa drei bis vier Stunden pro Woche nur mit dem Konflikt und nicht mit ihrer eigentlichen Arbeit. Auch besteht das Risiko, dass Patienten solche Streitigkeiten während ihrer Wartezeit mitbekommen und diese negativen Erfahrungen weitererzählen.

\section{Mitarbeiter wollen Struktur}

Viele Ärzte ärgern sich beispielsweise über das mangelnde Engagement einzelner Mitarbeiter in Teambesprechungen. Wenn Menschen in einer Gruppe an einem gemeinsamen Ziel arbeiten, reduziert sich häufig die Leistung des Einzelnen. Der Effekt tritt immer dann ein, wenn unklar ist, wie viel jeder zur Gesamtleistung beitragen soll. Dieses $\mathrm{Zu}$ rücklehnen in der „sozialen Hängematte" ist normal. Es ist kein Ausdruck von Unwillen einzelner Mitarbeiter. Gegensteuern lässt sich, indem neben Teamaufgaben auch individuelle Aufgaben verteilt werden, welche die Leistung des Einzelnen eben doch sichtbar machen. Wenn zum Beispiel als gemeinsames
Praxisprojekt geplant ist, die Patientenzufriedenheit über verbesserten Service und patientenorientierte Kommunikation zu erhöhen, dann sollten erst einzelne Bereiche wie Telefonservice, Wartezeiten oder Kommunikation mit anspruchsvollen Patienten zur Vorbereitung an einzelne Mitarbeiter gegeben werden. Erst im Anschluss daran wird alles mit dem gesamten Praxisteam weiterentwickelt. Bei diesem Vorgehen kann sich niemand zurückziehen, jeder Beitrag ist sichtbar.

\section{Der ideale Mitarbeiter - ein Mythos?}

Einen absolut idealen Mitarbeiter gibt es wohl nicht, aber es gibt die ideale Besetzung für einen bestimmten Arbeitsplatz. Bei der Besetzung einer Stelle sollte daher darauf geachtet werden, welche Stärken und Talente der Mitarbeiter mitbringt, und an welchem Arbeitsplatz er diese am besten einsetzen kann. Demgegenüber wird der Einsatz nach defizitorientierten Gesichtspunkten nach dem Motto „Das müssen Sie jetzt ganz besonders lernen“ eher einen negativen Effekt haben. Der entsprechende Mitarbeiter wird in diesem Bereich einfach nie so gut sein wie die Kollegen mit mehr Talent. Die Fehlerhäufigkeit steigt und Demotivation ist die Folge. Ein kontinuierliches Rotieren, wie es in vielen Praxen üblich ist, sollte daher gut überlegt sein. Denn dabei arbeiten Mitarbeiter immer wieder eine ziemlich lange Zeit auf Positionen, in denen sie eventuell talentfrei sind.

\section{Neue Mitarbeiter - was zählt?}

Die Praxismitarbeiter sind die wichtigste "Software“ einer Praxis. Ein großer Anteil des Eindrucks, den ein Patient aus der Praxis mitnimmt, resultiert nicht aus der Behandlung durch den Arzt, sondern aus dem Engagement der Mitarbeiter. Beim Bewerbungsgespräch lassen sich Ärzte häufig allein durch die fachliche Qualifikation eines Bewerbers beeindrucken. Weitaus seltener wird ein Blick auf die persönliche Eignung geworfen und diese systematisch in das Bewerbungsgespräch mit einbezogen.

Wenn ein Bewerber aber von seiner Persönlichkeit her nicht zu einem Praxisteam passt, kann dies später zu erheblicher Unzufriedenheit und sogar zur Kündigung führen. Bereits im Bewerbungsgespräch sollten daher Schlüsselqualifikationen wie Teamfähigkeit, Flexibilität, Kommunikations- und Kontaktfähigkeit abgefragt werden. Dafür können etwa einfache Situationen aus dem Praxisalltag als Fallbeispiele („Wie würden Sie reagieren, wenn ...“) hilfreich sein.

Die fachliche Eignung eines Mitarbeiters lässt sich nämlich im Nachhinein meist verbessern, die soziale Kompetenz und Persönlichkeit dagegen kaum.

\section{Dr. Andrea Schuhmacher}

Dr. Schuhmacher Consulting \& Training GmbH, Köln

Andrea Becker

Frielingsdorf Consult GmbH, Köln 